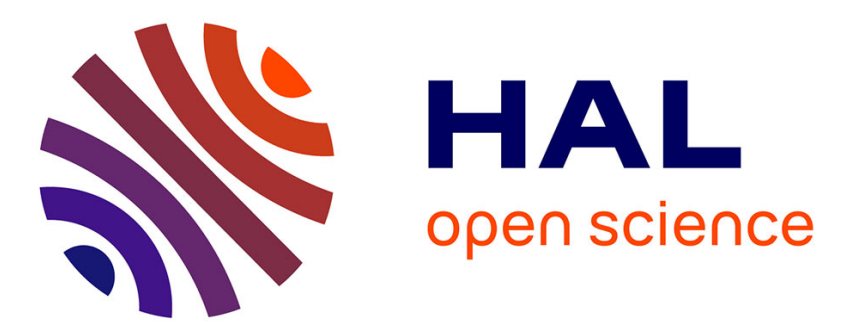

\title{
Fast multipole method applied to Symmetric Galerkin boundary element method for 3D elasticity and fracture problems
}

\author{
Anh Duc Pham, Saida Mouhoubi, Marc Bonnet, Cyrille Chazallon
}

\section{- To cite this version:}

Anh Duc Pham, Saida Mouhoubi, Marc Bonnet, Cyrille Chazallon. Fast multipole method applied to Symmetric Galerkin boundary element method for 3D elasticity and fracture problems. Engineering Analysis with Boundary Elements, 2012, 36, pp.1838-1847. 10.1016/j.enganabound.2012.07.004 . hal-00721705

\section{HAL Id: hal-00721705 \\ https://hal.science/hal-00721705}

Submitted on 30 Jul 2012

HAL is a multi-disciplinary open access archive for the deposit and dissemination of scientific research documents, whether they are published or not. The documents may come from teaching and research institutions in France or abroad, or from public or private research centers.
L'archive ouverte pluridisciplinaire HAL, est destinée au dépôt et à la diffusion de documents scientifiques de niveau recherche, publiés ou non, émanant des établissements d'enseignement et de recherche français ou étrangers, des laboratoires publics ou privés. 


\title{
Fast multipole method applied to Symmetric Galerkin boundary element method for 3D elasticity and fracture problems
}

\author{
Anh Duc PHAM ${ }^{\mathrm{a}}$, Saida MOUHOUBI ${ }^{\mathrm{a}}$, Marc BONNET ${ }^{\mathrm{b}, *}$, Cyrille CHAZALLON ${ }^{\mathrm{a}}$ \\ ${ }^{a}$ Laboratoire de Génie de la Conception, 24 boulevard de la victoire, 67084 Strasbourg Cedex, FRANCE \\ ${ }^{b}$ Laboratoire de Mécanique des Solides, École Polytechnique, 91128 Palaiseau Cedex, FRANCE
}

\begin{abstract}
The solution of three-dimensional elastostatic problems using the Symmetric Galerkin Boundary Element Method (SGBEM) gives rise to fully-populated (albeit symmetric) matrix equations, entailing high solution times for large models. This article is concerned with the formulation and implementation of a multi-level fast multipole SGBEM (FM-SGBEM) for elastic solid with cracks. Arbitrary geometries and boundary conditions may be considered. Numerical results on test problems involving a cube, single or multiple cracks in an unbounded medium, and a cracked cylindrical solid are presented. BEM models involving up to $10^{6} \mathrm{BEM}$ unknowns are considered, and the desirable predicted trends of the elastostatic FM-SGBEM, such as a $O(N)$ complexity per iteration, are verified.
\end{abstract}

Keywords: Fast Multipole Method (FMM), Elasticity, Symmetric Galerkin Boundary Element Method (SGBEM), Fracture Mechanics.

\section{Introduction}

The boundary element method $[5,7,10]$ (BEM), pioneered in the sixties [35, 16], is a mesh reduction method based on the discretization of boundary integral equations (BIEs). It is subject to restrictive constitutive assumptions but yields highly accurate solutions, and is in particular well suited to deal with unbounded-domain idealizations. Moreover, the surface-only mesh greatly facilitates data preparation for complex geometries.

Fracture mechanics problems are one of the main areas of applications of the BEM to solid mechanics, see e.g. the review article [1]. In such cases, cracks are directly represented as displacement discontinuity loci and the traction integral equation is employed to enforce static conditions on the crack faces and permit single-domain formulations irrespective of the number of cracks involved, see e.g. $[12,17]$ for early expositions of these ideas. However, traction collocation BIE formulations, whether employed in isolation or as a component of the so-called dual approach [30], involve an hypersingular kernel. This in turn requires $C^{1, \alpha}$ regularity of the density (usually the displacement discontinuity) at the collocation points and specially designed singular integration procedures, making the implementation of traction CBIEs quite involved. In contrast, the symmetric Galerkin BIE (SGBIE) approach to crack problems only requires $H^{1 / 2}$ densities for the continuous problem, making $C^{0}$ boundary element interpolations suitable (see [12] for an early implementation of the latter approach for plane cracks, and also $[21,22,19,8,28]$, among many references on this treatmnent). Moreover, SGBEM is based on a variational (weak) version of the integral equations, thus

\footnotetext{
${ }^{*}$ Corresponding author. Current address: POems (UMR 7231 CNRS), Appl. Math. Dept., ENSTA, Paris, France

Email addresses: saida.mouhoubi@insa-strasbourg.fr (Saida MOUHOUBI), mbonnet@ensta.fr (Marc BONNET)
}

Preprint submitted to Eng. Anal. Bound. Elem.

July 21, 2012 
entailing double integrations, and leads (through an appropriate discretization scheme) to matrix operators which exhibit symmetry and sign-definiteness.

The Symmetric Galerkin BEM (SGBEM) is known to yield symmetric but fully populated matrices, see e.g. [7] or the references in the survey article [9]. Letting $N$ denote the number of BEM unknowns, conventional solution methods for the SGBEM thus $O\left(N^{2} / 2\right)$ memory, $O\left(N^{2}\right)$ setup computing time and $O\left(N^{3} / 6\right)$ solution computing time using a direct solver. Domain methods, such as Finite Element Method (FEM), require $O\left(N_{\mathrm{FEM}}\right)$ set-up computing time and $O\left(N_{\mathrm{FEM}}\right)$ memory, with $N_{\text {FEM }}$ denoting the number of FEM unknowns (which is significantly larger than $N$ for a given configuration and accuracy).

The above-mentioned complexities are major bottlenecks that prevent the application of standard (SG)BEMs to large-scale problems. Faster strategies, i,e. treatments which reduce the abovequoted complexities and thereby allow to still exploit the known advantages of BEMs when large $N$ prohibits the use of traditional implementations, appeared around 1985 with an iterative integralequation approach for solving 2-D Laplace problems within $O(N)$ CPU time per iteration [36]. The fast multipole method (FMM) concept was introduced in [23], in the context of many-particle simulations. The FMM then naturally led to fast multipole boundary element methods (FMBEMs), whose scope and capabilities have rapidly progressed. FM-BEMs have since been used for many engineering problems involving e.g. Stokes flows [20, 18], acoustics [2, 43], electromagnetism [15], elastodynamics [14, 41, 13]. Applications of FM-BEMs to FEM-BEM coupling are found in e.g. [29, 11], while FM-BEMs for crack problems involving 3D unbounded bodies are addressed in [33] (Laplace equation) and [46, 45, 27] (elastostatics). More references can be found in the review article [32]. The FMM, as well as other fast BEM approaches [44, 25, 26, 42], intrinsically relies upon an iterative solution approach for the linear system of discretized BEM equations, with solution times typically of order $O(N)$ per iteration for static problems using the multi-level version of the FMM (instead of $O\left(N^{2}\right)$ per iteration with traditional forms of the BEM used in conjunction with iterative solvers).

FM-BEMs can aso be developed for SGBIE formulations, see e.g. [34] for potential problems, [40] for electromagnetic problems or [27] for cracks in unbounded media. This article is concerned with the formulation and implementation of a multi-level fast multipole SGBEM (FM-SGBEM) for elastic solid with cracks.

The article is organised as follows. The relevant SGBIE formulation is reviewed in Sec. 2. The fast multipole treatment for the elastostatic SGBEM is next described in Sec. 3. Numerical examples are then presented in Sec. 4, with test problems involving a cube (Sec. 4.1), single or multiple cracks in an unbounded medium (Secs. 4.2 and 4.3), and a cracked cylindrical solid (Sec. 4.4). BE models involving up to $N=10^{6}$ unknowns are considered, and the desirable predicted trends of the elastostatic FMM, such as a $O(N)$ complexity per iteration, are observed on the numerical results.

\section{Fundamentals of the 3D-SGBEM}

\subsection{Elastostatic problems}

Let us consider a three-dimensional elastic body $\Omega$, either bounded or unbounded. The stress state at the point $\boldsymbol{y}$ inside the body is described by the stress tensor $\boldsymbol{\sigma}$ with components $\sigma_{i j}$, the tractions relevant to a direction $\boldsymbol{n}$ being thus given by

$$
t_{i}(\boldsymbol{y})=\sigma_{i j} n_{j}(\boldsymbol{y}) \quad(i, j=1,2,3)
$$


with $\boldsymbol{n}$ denoting the outward unit normal to $\Omega$. In the absence of body forces, $\boldsymbol{\sigma}$ satisfies the equilibrium equations

$$
\sigma_{i j, j}(\boldsymbol{y})=0 \quad(\boldsymbol{y} \in \Omega)
$$

where (.),j stands for the derivative of (.) along the $j^{\text {th }}$ coordinate. In Eqs. (1), (2) and throughout this article, Einstein's convention of implicit summation over repeated indices is used.

The constitutive law for a linear elastic and isotropic material (Hooke's law) reads:

$$
\sigma_{i j}(\boldsymbol{y})=C_{i j h k} \varepsilon_{h k}(\boldsymbol{y})
$$

where the components $\varepsilon_{i j}$ of the linearized strain tensor are related to displacements by:

$$
\varepsilon_{i j}(\boldsymbol{y})=\frac{1}{2}\left[u_{i, j}(\boldsymbol{y})+u_{j, i}(\boldsymbol{y})\right]
$$

and the coefficients $C_{i j h k}$ of the fourth-order elasticity tensor $\mathcal{C}$ are given in terms of the Poisson ratio $\nu$ and shear modulus $\mu$ by

$$
C_{i j h k}=2 \mu\left[\frac{\nu}{1-2 \nu} \delta_{i j} \delta_{h k}+\delta_{i k} \delta_{j h}+\delta_{i h} \delta_{j k}\right] .
$$

(with $\delta_{i j}$ denoting the Kronecker symbol). The elastic solid is subjected to imposed boundary traction $\boldsymbol{t}=\boldsymbol{t}^{\mathrm{D}}$ on the surface $S_{T}$ and prescribed displacements $\boldsymbol{u}=\boldsymbol{u}^{\mathrm{D}}$ on $S_{u}\left(\partial \Omega=\bar{S}_{u} \cup \bar{S}_{T}\right.$, $S_{u} \cap S_{T}=\emptyset$ ), as sketched in Fig.1. The direct symmetric Galerkin BIE (SGBIE) formulation governing the unknown boundary traces $\boldsymbol{u}$ on $S_{T}$ and $\boldsymbol{t}$ on $S_{u}$ for this mixed elastostatic boundary value problem reads $[6,9,38]$ :

$$
\text { Find }(\boldsymbol{u}, \boldsymbol{t}) \in \mathcal{V}_{u} \times \mathcal{V}_{T},\left\{\begin{array}{l}
\mathcal{B}_{u u}(\boldsymbol{u}, \widetilde{\boldsymbol{u}})+\mathcal{B}_{t u}(\boldsymbol{t}, \widetilde{\boldsymbol{u}})=\mathcal{F}_{u}(\widetilde{\boldsymbol{u}}) \\
\mathcal{B}_{u t}(\boldsymbol{u}, \widetilde{\boldsymbol{t}})+\mathcal{B}_{t t}(\boldsymbol{t}, \widetilde{\boldsymbol{t}})=\mathcal{F}_{t}(\widetilde{\boldsymbol{t}})
\end{array} \quad \forall(\widetilde{\boldsymbol{u}}, \widetilde{\boldsymbol{t}}) \in \mathcal{V}_{u} \times \mathcal{V}_{T}\right.
$$

using the bilinear forms :

$$
\begin{aligned}
\mathcal{B}_{u u}(\boldsymbol{u}, \widetilde{\boldsymbol{u}}) & =\int_{S_{T}} \int_{S_{T}}[R u]_{i q}(\boldsymbol{x}) B_{i k q s}(\boldsymbol{r})[R \widetilde{u}]_{k s}(\tilde{\boldsymbol{x}}) \mathrm{d} S_{\tilde{x}} \mathrm{~d} S_{x} \\
\mathcal{B}_{t t}(\boldsymbol{t}, \widetilde{\boldsymbol{t}}) & =\int_{S_{u}} \int_{S_{u}} t_{k}(\boldsymbol{x}) U_{i}^{k}(\boldsymbol{x}, \tilde{\boldsymbol{x}}) \widetilde{t}_{i}(\tilde{\boldsymbol{x}}) \mathrm{d} S_{\tilde{x}} \mathrm{~d} S_{x} \\
\mathcal{B}_{t u}(\boldsymbol{t}, \widetilde{\boldsymbol{u}}) & =-\int_{S_{u}} \int_{S_{T}} t_{k}(\boldsymbol{x}) T_{i}^{k}(\boldsymbol{x}, \tilde{\boldsymbol{x}}) \widetilde{u}_{i}(\tilde{\boldsymbol{x}}) \mathrm{d} S_{\tilde{x}} \mathrm{~d} S_{x} \\
\mathcal{B}_{u t}(\boldsymbol{u}, \widetilde{\boldsymbol{t}}) & =\mathcal{B}_{t u}(\widetilde{\boldsymbol{t}}, \boldsymbol{u})
\end{aligned}
$$

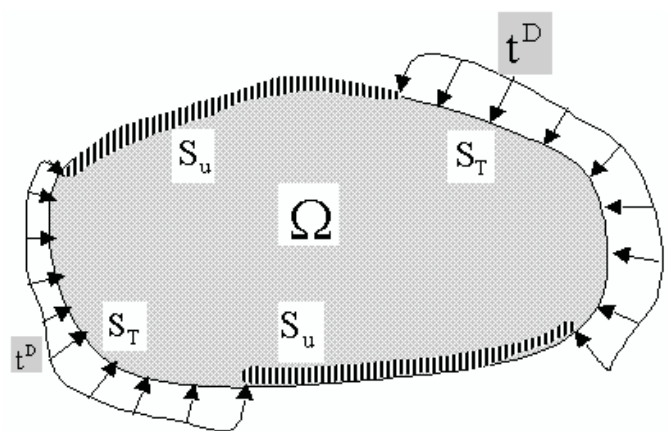

Figure 1: Elastic solid: notation 
and the linear forms:

$$
\begin{aligned}
\mathcal{F}_{u}(\widetilde{\boldsymbol{u}})= & \int_{S_{T}} \int_{S_{T}} t_{k}^{\mathrm{D}}(\boldsymbol{x}) T_{i}^{k}(\boldsymbol{x}, \tilde{\boldsymbol{x}})\left[\widetilde{u}_{i}(\tilde{\boldsymbol{x}})-\widetilde{u}_{i}(\boldsymbol{x})\right] \mathrm{d} S_{\tilde{x}} \mathrm{~d} S_{x}-\int_{S_{T}} \int_{S_{u}} t_{k}^{\mathrm{D}}(\boldsymbol{x}) T_{i}^{k}(\boldsymbol{x}, \tilde{\boldsymbol{x}}) \widetilde{u}_{i}(\boldsymbol{x}) \mathrm{d} S_{\tilde{x}} \mathrm{~d} S_{x} \\
& -\int_{S_{u}} \int_{S_{T}}[R u]_{i q}^{\mathrm{D}}(\boldsymbol{x}) B_{i k q s}(\boldsymbol{r})[R \widetilde{u}]_{k s}(\tilde{\boldsymbol{x}}) \mathrm{d} S_{\tilde{x}} \mathrm{~d} S_{x}+(\kappa-1) \int_{S_{T}} t_{k}^{\mathrm{D}}(\boldsymbol{x}) \widetilde{u}_{k}(\boldsymbol{x}) \mathrm{d} S_{x} \\
\mathcal{F}_{t}(\widetilde{\boldsymbol{t}})= & \int_{S_{u}} \int_{S_{u}}\left[u_{i}^{\mathrm{D}}(\boldsymbol{x})-u_{i}^{\mathrm{D}}(\tilde{\boldsymbol{x}})\right] T_{i}^{k}(\tilde{\boldsymbol{x}}, \boldsymbol{x}) \widetilde{t}_{k}(\tilde{\boldsymbol{x}}) \mathrm{d} S_{x} \mathrm{~d} S_{\tilde{x}}-\int_{S_{u}} \int_{S_{T}} u_{i}^{\mathrm{D}}(\tilde{\boldsymbol{x}}) T_{i}^{k}(\tilde{\boldsymbol{x}}, \boldsymbol{x}) \widetilde{t}_{k}(\tilde{\boldsymbol{x}}) \mathrm{d} S_{x} \mathrm{~d} S_{\tilde{x}} \\
& -\int_{S_{T}} \int_{S_{u}} t_{k}^{\mathrm{D}}(\boldsymbol{x}) U_{i}^{k}(\boldsymbol{x}, \tilde{\boldsymbol{x}}) \widetilde{t}_{i}(\tilde{\boldsymbol{x}}) \mathrm{d} S_{\tilde{x}} \mathrm{~d} S_{x}+\kappa \int_{S_{u}} u_{k}^{\mathrm{D}}(\boldsymbol{x}) \widetilde{t}_{k}(\boldsymbol{x}) \mathrm{d} S_{x}
\end{aligned}
$$

(where $\kappa=0$ or 1 depending on whether the unit normal to $S_{u}, S_{T}$ is directed towards the exterior or interior of that surface, respectively). In $(7)-(9), U_{i}^{k}(\boldsymbol{x}, \tilde{\boldsymbol{x}})$ and $T_{i}^{k}(\boldsymbol{x}, \tilde{\boldsymbol{x}})$ respectively denote the components in the direction $i$ of the Kelvin fundamental displacement and traction at $\boldsymbol{x} \in \mathbb{R}^{3}$ created in an elastic full-space by a point force applied at $\tilde{\boldsymbol{x}} \in \mathbb{R}^{3}$, and are given by

$$
\begin{aligned}
U_{k}^{i}(\boldsymbol{x}, \tilde{\boldsymbol{x}}) & =\frac{K}{r}\left[\hat{r}_{i} \hat{r}_{k}(3-4 \nu)+\delta_{i k}\right] \\
T_{i}^{k}(\boldsymbol{x}, \tilde{\boldsymbol{x}}) & =-\frac{2 \mu K}{r^{2}} n_{j}(\boldsymbol{x})\left[3 \hat{r}_{i} \hat{r}_{k} \hat{r}_{j}+(1-2 \nu)\left(\delta_{i k} \hat{r}_{j}+\delta_{j k} \hat{r}_{i}-\delta_{i j} \hat{r}_{k}\right)\right],
\end{aligned}
$$

having set

$$
\boldsymbol{r}=\boldsymbol{x}-\tilde{\boldsymbol{x}}, \quad r=\|\boldsymbol{r}\|, \quad \hat{\boldsymbol{r}}=\boldsymbol{r} / r
$$

and

$$
K=[16 \pi \mu(1-\nu)]^{-1}
$$

Moreover, the spaces $\mathcal{V}_{u}$ and $\mathcal{V}_{T}$ of admissible boundary traces of displacements and tractions are defined as

$$
\begin{aligned}
& \mathcal{V}_{u}=\left\{\boldsymbol{u} \in H^{1 / 2}(S), \operatorname{supp}(\boldsymbol{u}) \subset S_{T}\right\} \\
& \mathcal{V}_{T}=\left\{\boldsymbol{u} \in H^{-1 / 2}(S), \operatorname{supp}(\boldsymbol{t}) \subset \bar{S}_{u}\right\},
\end{aligned}
$$

and $\widetilde{\boldsymbol{u}}, \widetilde{\boldsymbol{t}}$ are test displacements and tractions. Natural finite-dimensional subspaces of $\mathcal{V}_{u}$ and $\mathcal{V}_{T}$ for Galerkin discretization consist of continuous interpolations of $\boldsymbol{u}$ over $S_{T}$ with a zero trace on the edge $\partial S_{T}$ and piecewise-continuous interpolation of $\boldsymbol{t}$ over $S_{u}$. In particular, in contrast to the case of the traction CBIE, the interpolation method puts no requirement on the derivatives of $\boldsymbol{u}$. Note that the data $\boldsymbol{u}^{\mathrm{D}}$ appearing in (8) and (9) is actually an arbitrary extension to $\partial \Omega$ of the displacement value prescribed on $S_{u}$, having $\boldsymbol{u}^{\mathrm{D}} \in H^{1 / 2}(\partial \Omega)$ regularity, so that the actual displacement on $S_{T}$ is $\boldsymbol{u}+\boldsymbol{u}^{\mathrm{D}}$. This allows $\boldsymbol{u}$ and $\widetilde{\boldsymbol{u}}$ to belong to the same space $\mathcal{V}_{u}$, and also explains that (9) involves $\boldsymbol{u}^{\mathrm{D}}$ in an integral over $S_{T}$.

Formulation (7)-(9) is written in regularized form [6,31], which involves only weakly singular double surface integrals with $O\left(r^{-1}\right)$ integrands. The regularization procedure yielding (7)-(9) involves the Stokes theorem together with indirect regularization. The surface curl operator $R$ arising as a result of this manipulation, introduced e.g in $[31,4]$, is defined by $[R u]_{k s}(\tilde{\boldsymbol{x}})=e_{j r s} n_{j} u_{k, r}(\boldsymbol{y})$ (where $e_{j r s}$ denotes the permutation symbol), while the weakly singular fourth-order tensor $B_{i k q s}$ is given by

$$
B_{i k q s}(\boldsymbol{r})=\frac{2 \mu K}{r}\left[2 \delta_{q s} \hat{r}_{i} \hat{r}_{k}+2\left(\delta_{i k} \delta_{q s}-2 \nu \delta_{i s} \delta_{k q}-(1-\nu) \delta_{i q} \delta_{k s}\right)\right]
$$




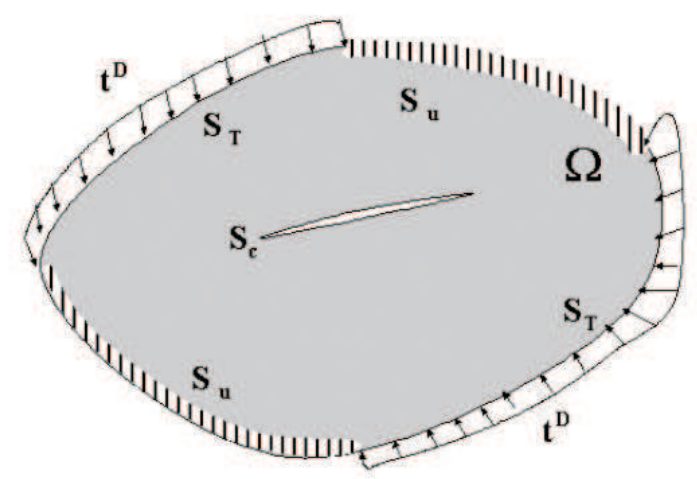

Figure 2: 3D linear elastic cracked domain

\subsection{Crack problems}

The previously discussed elastic body now contains a crack (or a set thereof) embedded inside $\Omega$ (Fig. 2), conceived as an open (possibly non connected) surface $S_{c}$ which is the locus of displacement discontinuity $\phi$, defined by

$$
\phi(x):=\boldsymbol{u}\left(\boldsymbol{x}^{+}\right)-\boldsymbol{u}\left(\boldsymbol{x}^{-}\right)
$$

(with $\boldsymbol{x}^{+} \in S_{c}^{+}$and $\boldsymbol{x}^{-} \in S_{c}^{-}, S_{c}^{+}$and $S_{c}^{-}$being the upper and lower crack faces) and generated by the application of equal and opposite tractions on $S_{c}$ :

$$
\boldsymbol{p}(\boldsymbol{x}) \equiv \boldsymbol{p}\left(\boldsymbol{x}^{-}\right)=-\boldsymbol{p}\left(\boldsymbol{x}^{+}\right)
$$

(the unit normal to $S_{c}$ being conventionally directed from $S_{c}^{-}$to $S_{c}^{+}$), i.e. $\boldsymbol{n}:=\boldsymbol{n}^{-}=-\boldsymbol{n}^{+}$). The boundary of $\Omega$ is now $\partial \Omega=S_{c} \cup S$, with the external surface $S$ split into $S=S_{u} \cup S_{T}$.

The direct SGBIE formulation for the mixed elastostatic boundary value problem for a cracked solid is also well-documented in the literature and can be established in many ways, see e.g. [19]. For instance, one can complete $S_{c}$ with a fictitious surface $\hat{S}_{c} \subset \Omega$ so that the closure of $S_{c} \cup \hat{S}_{c}$ is a closed surface enclosing a subdomain $\Omega^{-}$of $\Omega$. Defining the complementary subregion $\Omega^{+}=\Omega \backslash \overline{\Omega^{-}}$, one may then write the SGBIE formulation (6) for each subregion $\Omega^{+}$and $\Omega^{-}$, assuming equal and opposite prescribed tractions on $S_{c} \cup \hat{S}_{c}$ for both subregions and equal unknown and trial displacements on $\hat{S}_{c}$. Upon combining the resulting SGBIE formulations and invoking the abovestated continuity assumptions, one obtains:

Find $(\boldsymbol{u}, \boldsymbol{t}, \boldsymbol{\phi}) \in \mathcal{V}_{u} \times \mathcal{V}_{T} \times \mathcal{V}_{c}$,

$$
\left\{\begin{array}{rl}
\mathcal{B}_{u u}(\boldsymbol{u}, \widetilde{\boldsymbol{u}})+\mathcal{B}_{t u}(\boldsymbol{t}, \widetilde{\boldsymbol{u}})+\mathcal{B}_{\phi u}(\phi, \widetilde{\boldsymbol{u}}) & =\mathcal{F}_{u}(\widetilde{\boldsymbol{u}}) \\
\mathcal{B}_{u t}(\boldsymbol{u}, \widetilde{\boldsymbol{t}})+\mathcal{B}_{t t}(\boldsymbol{t}, \widetilde{\boldsymbol{t}})+\mathcal{B}_{\phi t}(\phi, \widetilde{\boldsymbol{t}}) & =\mathcal{F}_{t}(\widetilde{\boldsymbol{t}}) \\
\mathcal{B}_{u \phi}(\boldsymbol{u}, \widetilde{\boldsymbol{\phi}})+\mathcal{B}_{t \phi}(\boldsymbol{t}, \widetilde{\boldsymbol{\phi}})+\mathcal{B}_{\phi \phi}(\boldsymbol{\phi}, \widetilde{\boldsymbol{\phi}}) & =\mathcal{F}_{\phi}(\widetilde{\boldsymbol{\phi}})
\end{array} \quad \forall(\widetilde{\boldsymbol{u}}, \widetilde{\boldsymbol{t}}, \widetilde{\boldsymbol{\phi}}) \in \mathcal{V}_{u} \times \mathcal{V}_{T} \times \mathcal{V}_{c}\right.
$$

using the bilinear forms (7) and

$$
\begin{aligned}
\mathcal{B}_{\phi \phi}(\boldsymbol{\phi}, \widetilde{\boldsymbol{\phi}}) & =\int_{S_{c}} \int_{S_{c}}[R \phi]_{i q}(\boldsymbol{x}) B_{i k q s}(\boldsymbol{r})[R] \phi_{k s}(\tilde{\boldsymbol{x}}) \mathrm{d} S_{\tilde{x}} \mathrm{~d} S_{x} \\
\mathcal{B}_{\phi u}(\boldsymbol{\phi}, \widetilde{\boldsymbol{u}}) & =-\int_{S_{c}} \int_{S_{T}}[R \phi]_{i q}(\boldsymbol{x}) B_{i k q s}(\boldsymbol{r})[R \widetilde{u}]_{k s}(\tilde{\boldsymbol{x}}) \mathrm{d} S_{\tilde{x}} \mathrm{~d} S_{x}
\end{aligned}
$$




$$
\begin{aligned}
\mathcal{B}_{\phi t}(\boldsymbol{\phi}, \widetilde{\boldsymbol{t}}) & =\int_{S_{c}} \int_{S_{u}} T_{i}^{k}(\tilde{\boldsymbol{x}}, \boldsymbol{x}) \phi_{i}(\boldsymbol{x}) \widetilde{t}_{k}(\tilde{\boldsymbol{x}}) \mathrm{d} S_{x} \mathrm{~d} S_{\tilde{x}} \\
\mathcal{B}_{u \phi}(\boldsymbol{u}, \widetilde{\boldsymbol{\phi}}) & =\mathcal{B}_{\phi u}(\widetilde{\boldsymbol{\phi}}, \boldsymbol{u}) \\
\mathcal{B}_{t \phi}(\boldsymbol{t}, \widetilde{\boldsymbol{\phi}}) & =\mathcal{B}_{t \phi}(\widetilde{\boldsymbol{\phi}}, \boldsymbol{u})
\end{aligned}
$$

and the linear forms (8), (9) and

$$
\begin{aligned}
\mathcal{F}_{\phi}(\widetilde{\boldsymbol{\phi}})=-\int_{S_{u}} \int_{S_{c}}\left[R u^{\mathrm{D}}\right]_{i q}(\boldsymbol{x}) & B_{i k q s}(\boldsymbol{r})[R \widetilde{u}]_{k s}(\tilde{\boldsymbol{x}}) \mathrm{d} S_{\tilde{x}} \mathrm{~d} S_{x} \\
& \quad+\int_{S_{T}} \int_{S_{c}} t_{k}^{\mathrm{D}}(\boldsymbol{x}) T_{i}^{k}(\boldsymbol{x}, \tilde{\boldsymbol{x}}) \widetilde{u}_{i}(\tilde{\boldsymbol{x}}) \mathrm{d} S_{\tilde{x}} \mathrm{~d} S_{x}+\int_{S_{c}} p_{k}(\boldsymbol{x}) \widetilde{\phi}_{k}(\boldsymbol{x}) \mathrm{d} S_{x}
\end{aligned}
$$

In (16), the spaces of admissible boundary traces are $\mathcal{V}_{u}$ and $\mathcal{V}_{T}$, again defined by (14), and $\mathcal{V}_{c}=H_{0}^{1 / 2}\left(S_{c}\right)$. Natural finite-dimensional subspaces of $\mathcal{V}_{c}$ for Galerkin discretization then consist of continuous interpolations of $\phi$ over $S_{c}$ with a zero trace on the crack front $\partial S_{c}$, with again no requirement on the derivatives of $\phi$.

\subsection{Solution strategy for the SGBEM equations}

The classical SGBEM discretization approach for problems (7) or (16) is based on $C^{0}$ interpolations of $(\boldsymbol{u}, \widetilde{\boldsymbol{u}})$ over $S_{T}$ and $(\boldsymbol{\phi}, \widetilde{\boldsymbol{\phi}}) S_{c}$ and element-wise continuous interpolation of $(\boldsymbol{t}, \widetilde{\boldsymbol{t}})$ over $S_{u}$. The discretized set of equations thus obtained is symmetric by virtue of the fact that the same interpolation spaces are used for the unknowns and trial functions. It has the generic form

$$
[B]\{X\}=\{F\}
$$

where $[B] \in \mathbb{R}_{\mathrm{sym}}^{N \times N}$ is the symmetric matrix gathering the discretized versions of the bilinear forms, $\{X\} \in \mathbb{R}^{N}$ gathering all unknown DOFs on $S_{T}, S_{c}$ and $S_{u}$, and $\{F\} \in \mathbb{R}^{N}$ is the discretized version of the right-hand side of (7) or (16).

The influence matrix $[B]$ being fully-populated, storing it is limited, on ordinary computers, to BEM models of size not exceeding $N=O\left(10^{4}\right)$. Direct solvers for (19) require $O\left(N^{3}\right)$ arithmetic operations (i.e. have a $O\left(N^{3}\right)$ complexity), and are thus also limited to moderately-sized BEM models. BEM problems of larger size are preferably solved by means of iterative algorithms such as GMRES [37]. With reference to (19), each GMRES iteration requires one evaluation of $[B]\{X\}$ for given $\{X\}$, a task requiring $O\left(N^{2}\right)$ arithmetic operations regardless of whether $[B]$ is build and stored a priori, or $[B]\{X\}$ is evaluated by means of standard SGBEM numerical integration procedures for each GMRES iteration. In the latter case, the $O\left(N^{2}\right)$ complexity stems from the fact that, again, all double element integrals must be computed. Applications of the BEM to models significantly larger than $N=O\left(10^{4}\right)$ require a faster (i.e. of complexity less than $O\left(N^{2}\right)$ ) evaluation procedure for $[B]\{X\}$ that avoids explicit set-up and storage of $[B]$. The fast multipole method (FMM) fulfills these objectives.

\section{Fast Multipole Method for elastostatic SGBEM}

The FMM is based on a reformulation of the kernels $U_{i}^{k}, T_{i}^{k}, B_{i k q s}$ into multipole series, which achieve a separation of the variables $\boldsymbol{x}$ and $\tilde{\boldsymbol{x}}$. For this purpose, the relative position vector $\boldsymbol{r}=\boldsymbol{x}-\tilde{\boldsymbol{x}}$ 


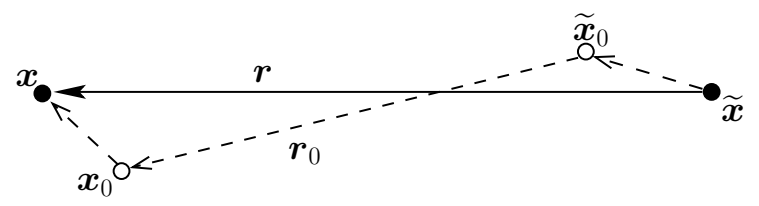

Figure 3: Decomposition of the position vector : notation

is decomposed into $\boldsymbol{r}=\boldsymbol{x}^{\prime}+\boldsymbol{r}_{0}-\tilde{\boldsymbol{x}}^{\prime}$, with $\boldsymbol{r}_{0}=\boldsymbol{x}_{0}-\tilde{\boldsymbol{x}}_{0}, \boldsymbol{x}^{\prime}=\boldsymbol{x}-\boldsymbol{x}_{0}$ and $\tilde{\boldsymbol{x}}^{\prime}=\tilde{\boldsymbol{x}}-\tilde{\boldsymbol{x}}_{0}$ in terms of two poles $\boldsymbol{x}_{0}, \tilde{\boldsymbol{x}}_{0}$. With these notations, the multipole expansion of $1 / r=1 /\|\boldsymbol{r}\|$ is given [45] by

$$
\begin{aligned}
\frac{1}{r} & =\sum_{n=0}^{\infty} \sum_{m=-n}^{n}(-1)^{n} R_{n m}\left(\tilde{\boldsymbol{x}}^{\prime}\right) \sum_{n^{\prime}=0}^{n} \sum_{m^{\prime}=-n^{\prime}}^{n^{\prime}} \overline{S_{n+n^{\prime}, m+m^{\prime}}\left(\boldsymbol{r}_{0}\right)} R_{n^{\prime} m^{\prime}}\left(\boldsymbol{x}^{\prime}\right) \\
R_{n m}(\boldsymbol{y}) & =\frac{1}{(n+m) !} P_{n}^{m}(\cos \alpha) e^{i m \beta} \rho^{n} \\
S_{n m}(\boldsymbol{y}) & =(n-m) ! P_{n}^{m}(\cos \alpha) e^{i m \beta} \rho^{-(n+1)}
\end{aligned}
$$

$(\rho, \alpha, \beta)$ being the spherical coordinates of the argument $\boldsymbol{y}$ and $P_{n}^{m}$ denoting the Legendre polynomials, and with the overbar denoting complex conjugation. The (complex-valued) solid harmonics $R_{n m}(\boldsymbol{y}), S_{n m}(\boldsymbol{y})$ can in fact be evaluated without actual recourse to spherical coordinates by means of the recursive formulae (A.1) to (A.5) initially proposed in [45]. For given poles $\boldsymbol{x}_{0}, \tilde{\boldsymbol{x}}_{0}$, expansion (20) is convergent for any $\boldsymbol{x}, \tilde{\boldsymbol{x}}$ such that

$$
\left\|\boldsymbol{x}^{\prime}\right\|<\left\|\tilde{\boldsymbol{x}}^{\prime}-\boldsymbol{r}_{0}\right\| \text { and }\left\|\tilde{\boldsymbol{x}}^{\prime}\right\|<\left\|\boldsymbol{x}^{\prime}+\boldsymbol{r}_{0}\right\| .
$$

It is then convenient to rewrite the Kelvin solution (11) in the form

$$
U_{k}^{i}(\boldsymbol{x}, \tilde{\boldsymbol{x}})=K\left[\delta_{i k}+(3-4 \nu)\left(x_{k}+r_{0 k}-\tilde{x}_{k}\right) \frac{\partial}{\partial \tilde{x}_{i}}\right] \frac{1}{r}
$$

which allows to take advantage of (20) for deriving a multipole expansion of $U_{k}^{i}$. The multipole expansion of $T_{k}^{i}$ can then found by means of a straightforward differentiation of the expansion of $U_{k}^{i}$, while $B_{i k q s}$ can be rewritten in a form similar to $(22)$.

Now, let $\Gamma\left(\boldsymbol{x}_{0}\right) \subset \partial \Omega$ and $\tilde{\Gamma}\left(\tilde{\boldsymbol{x}}_{0}\right) \subset \partial \Omega$ denote two subsets of $\partial \Omega$ such that (21) holds for any $\boldsymbol{x} \in \Gamma\left(\boldsymbol{x}_{0}\right)$ and $\tilde{\boldsymbol{x}} \in \tilde{\Gamma}\left(\tilde{\boldsymbol{x}}_{0}\right)$ (such subsets are said to be well-separated). Then, the contribution of surfaces $\Gamma\left(\boldsymbol{x}_{0}\right), \tilde{\Gamma}\left(\tilde{\boldsymbol{x}}_{0}\right)$ to the bilinear form $\mathcal{B}_{t t}(\boldsymbol{t}, \widetilde{\boldsymbol{t}})$, denoted by $B_{t t}\left(\boldsymbol{x}_{0}, \tilde{\boldsymbol{x}}_{0}\right)$ and given by

$$
B_{t t}\left(\boldsymbol{x}_{0}, \tilde{\boldsymbol{x}}_{0}\right):=\int_{\Gamma\left(\boldsymbol{x}_{0}\right)} \int_{\tilde{\Gamma}\left(\tilde{\boldsymbol{x}}_{0}\right)} t_{k}(\boldsymbol{x}) U_{i}^{k}(\boldsymbol{x}, \tilde{\boldsymbol{x}}) \widetilde{t}_{i}(\tilde{\boldsymbol{x}}) \mathrm{d} S_{\tilde{x}} \mathrm{~d} S_{x},
$$

can be evaluated by replacing the kernel $U_{k}^{i}$ by its multipole expansion, and likewise for the other bilinear forms involved in (6) or (16). For simplicity, only the multipole contribution $B_{t t}\left(\boldsymbol{x}_{0}, \tilde{\boldsymbol{x}}_{0}\right)$ is detailed next, the treatment of the other bilinear forms following the same approach.

Inserting expansion (20) into (22) and exploiting the resulting expression of $U_{i}^{k}$ in (23), the contribution $B_{t t}\left(\boldsymbol{x}_{0}, \tilde{\boldsymbol{x}}_{0}\right)$ to $\mathcal{B}_{t t}(\boldsymbol{t}, \widetilde{\boldsymbol{t}})$ is found to be given by

$$
\begin{aligned}
B_{t t}\left(\boldsymbol{x}_{0}, \tilde{\boldsymbol{x}}_{0}\right) & =\sum_{n=0}^{\infty} \sum_{m=-n}^{n}(-1)^{n} \sum_{n^{\prime}=0}^{n} \sum_{m^{\prime}=-n^{\prime}}^{n^{\prime}}\left\{\tilde{M}_{k n m}^{1}\left(\tilde{\boldsymbol{x}}_{0}\right) \overline{S_{n+n^{\prime}, m+m^{\prime}}\left(\boldsymbol{r}_{0}\right)} M_{k n^{\prime} m^{\prime}}^{1}\left(\boldsymbol{x}_{0}\right)\right. \\
& \left.+\tilde{M}_{k n m}^{1}\left(\tilde{\boldsymbol{x}}_{0}\right) r_{0 k} \overline{S_{n+n^{\prime}, m+m^{\prime}}\left(\boldsymbol{r}_{0}\right)} M_{n^{\prime} m^{\prime}}^{2}\left(\boldsymbol{x}_{0}\right)+\tilde{M}_{n m}^{2}\left(\tilde{\boldsymbol{x}}_{0}\right) \overline{S_{n+n^{\prime}, m+m^{\prime}}\left(\boldsymbol{r}_{0}\right)} M_{n^{\prime} m^{\prime}}^{2}\left(\boldsymbol{x}_{0}\right)\right\}
\end{aligned}
$$


in terms of the multipole moments

$$
\begin{aligned}
M_{k n m}^{1}\left(\boldsymbol{x}_{0}\right) & =\int_{S_{u}} R_{n m}\left(\boldsymbol{x}^{\prime}\right) t_{k}\left(\boldsymbol{x}^{\prime}\right) \mathrm{d} S_{x}^{\prime} \\
M_{n m}^{2}\left(\boldsymbol{x}_{0}\right) & =\int_{S_{u}} R_{n m}\left(\boldsymbol{x}^{\prime}\right) x_{k}^{\prime} t_{k}\left(\boldsymbol{x}^{\prime}\right) \mathrm{d} S_{x}^{\prime}
\end{aligned}
$$

associated to the pole $\boldsymbol{x}_{0}$ and

$$
\begin{aligned}
\tilde{M}_{k n m}^{1}\left(\tilde{\boldsymbol{x}}_{0}\right) & =\int_{S_{u}}\left[\delta_{i k}-(3-4 \nu) \tilde{x}_{k} \frac{\partial}{\partial \tilde{x}_{i}}\right] R_{n m}\left(\tilde{\boldsymbol{x}}^{\prime}\right) \widetilde{t}_{i}\left(\boldsymbol{x}^{\prime}\right) \mathrm{d} S_{\tilde{x}}^{\prime} \\
\tilde{M}_{n m}^{2}\left(\tilde{\boldsymbol{x}}_{0}\right) & =(3-4 \nu) \int_{S_{u}} \frac{\partial}{\partial \tilde{x}_{i}} R_{n m}\left(\tilde{\boldsymbol{x}}^{\prime}\right) \widetilde{t}_{i}\left(\tilde{\boldsymbol{x}}^{\prime}\right) \mathrm{d} S_{\tilde{x}}^{\prime}
\end{aligned}
$$

associated to the pole $\tilde{\boldsymbol{x}}_{0}$. Equation (24) can then be recast into the following equivalent form

$$
\mathcal{B}_{t t}(\boldsymbol{t}, \widetilde{\boldsymbol{t}})=\sum_{n=0}^{\infty} \sum_{m=-n}^{n}(-1)^{n}\left\{\tilde{M}_{k n m}^{1}\left(\tilde{\boldsymbol{x}}_{0}\right) L_{k n m}^{1}\left(\tilde{\boldsymbol{x}}_{0}\right)+\tilde{M}_{n m}^{2}\left(\tilde{\boldsymbol{x}}_{0}\right) L_{n m}^{2}\left(\tilde{\boldsymbol{x}}_{0}\right)\right\}
$$

in terms of the local expansion coefficients, related to the multipole moments by the following multipole-to-local (M2L) relations:

$$
\begin{aligned}
L_{k n m}^{1}\left(\boldsymbol{x}_{0}\right) & =\sum_{n^{\prime}=0}^{n} \sum_{m^{\prime}=-n^{\prime}}^{n^{\prime}} \overline{S_{n+n^{\prime}, m+m^{\prime}}\left(\boldsymbol{r}_{0}\right)}\left[M_{k n^{\prime} m^{\prime}}^{1}\left(\boldsymbol{x}_{0}\right)+r_{0 k} M_{n^{\prime} m^{\prime}}^{2}\left(\boldsymbol{x}_{0}\right)\right] \\
L_{n m}^{2}\left(\boldsymbol{x}_{0}\right) & =\sum_{n^{\prime}=0}^{n} \sum_{m^{\prime}=-n^{\prime}}^{n^{\prime}} \overline{S_{n+n^{\prime}, m+m^{\prime}}\left(\boldsymbol{r}_{0}\right)} M_{n^{\prime} m^{\prime}}^{2}\left(\boldsymbol{x}_{0}\right)
\end{aligned}
$$

\subsection{Outline of the FM-SGBEM algorithm}

Traditional methods for evaluating the left-hand side (i.e. the matrix-vector product $[B]\{X\}$ after discretization) of the SGBIE formulation (16) require that all double surface integrals over Cartesian products of elements be computed, leading to a $O\left(N^{2}\right)$ complexity. In contrast, equation (24) shows that the set of double surface integrals corresponding to two clusters of elements lying in $\Gamma\left(\boldsymbol{x}_{0}\right)$ and $\tilde{\Gamma}\left(\tilde{\boldsymbol{x}}_{0}\right)$ is replaced with a limited number of interactions between multipole moments given by single integrals over the elements in $\Gamma\left(\boldsymbol{x}_{0}\right)$ and $\tilde{\Gamma}\left(\tilde{\boldsymbol{x}}_{0}\right)$. This of course relies crucially on the fact that the series (20), and hence also (27) and the analogous series for the other bilinear and linear operators, are in practice truncated, the summation stopping at $n=p$, with $p=5-10$ usually yielding sufficient accuracy.

To optimize the acceleration permitted by (27), a hierarchical oct-tree structure of elements is introduced. For that purpose, a cube containing the whole boundary $\partial \Omega$, called 'level-0 cell', is divided into eight cubes (level- 1 cells), each of which is divided in the same fashion. A level- $\ell$ cell is divided into level- $(\ell+1)$ cells unless it contains less than a preset (relatively small) number $L$ of boundary elements ( $L$ is termed thereafter the subdivision-stopping parameter). Such cells are referred to as leaves, and the leaf level is denoted $\bar{\ell}$. Any given boundary element is deemed to belong to one cell of a given level only, even if is geometrically shared by two or more samelevel cells. Denoting by $\mathcal{C}^{(\ell)}\left(\boldsymbol{x}_{0}^{(\ell)}\right)$ a generic level- $\ell$ cubic cell centered at $\boldsymbol{x}_{0}^{(\ell)}$, the multi-level 
FMM $[32,24]$ basically consists in a systematic exploitation of the reformulation (27) of $(27)$ with $\Gamma\left(\boldsymbol{x}_{0}\right)=\mathcal{C}^{(\ell)}\left(\boldsymbol{x}_{0}^{(\ell)}\right)$ and $\tilde{\Gamma}\left(\tilde{\boldsymbol{x}}_{0}\right)=\mathcal{C}^{(\ell)}\left(\tilde{\boldsymbol{x}}_{0}^{(\ell)}\right)$ whenever the cells $\mathcal{C}^{(\ell)}\left(\boldsymbol{x}_{0}^{(\ell)}\right)$ and $\mathcal{C}^{(\ell)}\left(\tilde{\boldsymbol{x}}_{0}^{(\ell)}\right)$ are not adjacent, starting from the highest level 2 (i.e. the largest cells) and tracing the levels downward to accommodate contributions from pairs of smaller non-adjacent cells whose parents are adjacent (and thus cannot be accommodated at the levels above the current one. Interactions (27) that are left out by this process are the near-field interactions such that $\Gamma\left(\boldsymbol{x}_{0}\right)$ and $\tilde{\Gamma}\left(\tilde{\boldsymbol{x}}_{0}\right)$ belong to adjacent (or identical) cells at any level. The main steps of the algorithm thus consists of:

(i) An upward pass: multipole moments (25) are first computed for the lowest-level cells and then recursively aggregated by moving upward in the tree until level 2 (i.e. the highest level which features non-adjacent cells) is reached. This operation requires "multipole-to-multipole" (M2M) identities to shift the origin from the center $\boldsymbol{x}_{0}^{(\ell+1}$ of a level- $(\ell+1)$ cell to the center $\boldsymbol{x}_{0}^{(\ell)}$ of a level- $\ell$ cell. The M2M identities are obtained by invoking the following property of the $R_{n m}$ :

$$
R_{n m}\left(\boldsymbol{x}^{(\ell)}\right)=\sum_{n^{\prime}=0}^{n} \sum_{m^{\prime}=-n^{\prime}}^{n^{\prime}} R_{n^{\prime} m^{\prime}}\left(\boldsymbol{x}_{0}^{(\ell+1)}-\boldsymbol{x}_{0}^{(\ell)}\right) R_{n-n^{\prime}, m-m^{\prime}}\left(\boldsymbol{x}^{(\ell+1)}\right),
$$

with $\boldsymbol{x}^{(\ell)}$ and $\boldsymbol{x}^{(\ell+1)}$ denoting the local position vectors relative to origins $\boldsymbol{x}_{0}^{(\ell)}$ and $\boldsymbol{x}_{0}^{(\ell+1}$ ), respectively, in (25), from which the $M_{k n m}^{1}\left(\boldsymbol{x}_{0}^{(\ell)}\right), M_{n m}^{2}\left(\boldsymbol{x}_{0}^{(\ell)}\right)$ can be expressed in terms of the $M_{k n m}^{1}\left(\boldsymbol{x}_{0}^{(\ell+1)}\right)$ and $M_{n m}^{2}\left(\boldsymbol{x}_{0}^{(\ell+1)}\right)$.

(ii) $A$ downward pass: local expansions are first computed at level $\ell=2$ and then evaluated at selected lower-level cells by tracing downwards the tree structure. This operation requires "local-to-local" (L2L) identities to shift the origin from the center $\tilde{\boldsymbol{x}}_{0}^{(\ell)}$ of a level- $\ell$ cell to the center $\tilde{\boldsymbol{x}}_{0}^{(\ell+1}$ of a level- $(\ell+1)$ cell. The L2L identities are obtained by (i) invoking property (30) written with levels $\ell$ and $(\ell+1)$ swapped, (ii) inserting the resulting identities in (26), from which the $M_{k n m}^{1}\left(\tilde{\boldsymbol{x}}_{0}^{(\ell+1)}\right), M_{n m}^{2}\left(\tilde{\boldsymbol{x}}_{0}^{(\ell+1)}\right)$ can be expressed in terms of the $M_{k n m}^{1}\left(\tilde{\boldsymbol{x}}_{0}^{(\ell)}\right), M_{n m}^{2}\left(\tilde{\boldsymbol{x}}_{0}^{(\ell)}\right)$, and (iii) using the obtained relationships in (27) and identifying the $L_{k n^{\prime} m^{\prime}}^{1}\left(\tilde{\boldsymbol{x}}_{0}^{(\ell+1)}\right), L_{n^{\prime} m^{\prime}}^{2}\left(\tilde{\boldsymbol{x}}_{0}^{(\ell+1)}\right)$ expressed in terms of the $L_{k n^{\prime} m^{\prime}}^{1}\left(\tilde{\boldsymbol{x}}_{0}^{(\ell)}\right), L_{n^{\prime} m^{\prime}}^{2}\left(\tilde{\boldsymbol{x}}_{0}^{(\ell)}\right)$ as the cofactors of $M_{k n m}^{1}\left(\tilde{\boldsymbol{x}}_{0}^{(\ell+1)}\right), M_{n m}^{2}\left(\tilde{\boldsymbol{x}}_{0}^{(\ell+1)}\right)$.

(iii) $A$ direct calculation: all remaining double element integrals (corresponding to pairs of elements that are not well-separated at any level) are evaluated using conventional (singular or non-singular) integration methods. all singular integrations are in particular handled in this step. For this task, there is a choice between (i) assembling all near-field contributions once and for all into a sparse matrix $\left[B_{\text {near }}\right]$ (which contains $O(N)$ entries) and then computing (sparse) matrixvector products, or (ii) recomputing directly at each GMRES iteration the near-field contribution to the matrix-vector product without assembling the near-field sparse matrix; method (i) is adopted here.

Each matrix-vector product evaluation requires (for static problems such as those considered here) a $O(N)$ computational effort using the above-described fast multipole SGBEM (FMSGBEM), instead of $O\left(N^{2}\right)$ using non-multipole SGBEM methods. For a fixed subdivisionstopping parameter $L$, the sparse matrix $\left[B_{\text {near }}\right]$ requires a $O(N)$ memory, while SGBEM with a restart parameter $m$ needs $O(m N)$ memory, so that the overall memory required by the FMSGBEM is of order $O(N)$. 


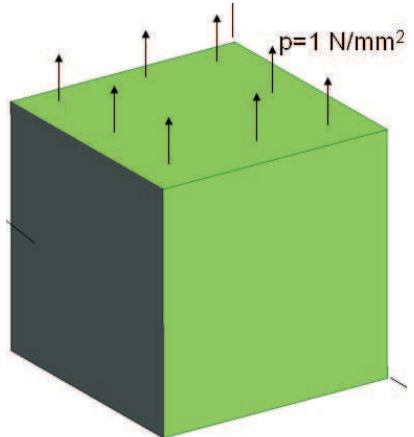

(a)

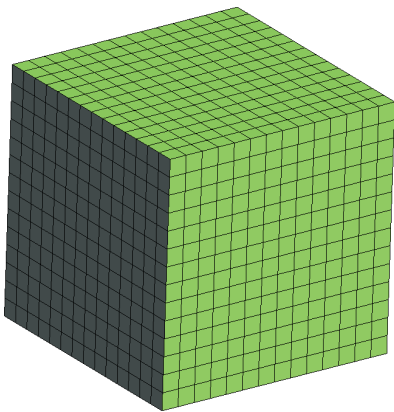

(b)

Figure 4: Clamped cube under uniaxial load: model (a) and mesh with 1176 elements (b)

\subsection{Main settings of the FM-SGBEM algorithm}

The proposed treatment has been implemented in Fortran 2008. On the basis of preliminary parameter studies, the truncation level $n=p$ in all multipole expansions such as (20) or (27) is set to $p=7$, while the subdivision-stopping parameter is chosen as $L=40$. The stopping criterion for GMRES is set as a predetermined $10^{-5}$ relative tolerance on the Euclidean norm of the residual. GMRES is used without preconditioning, and with the restart parameter set to $m=50$.

\section{Numerial examples}

The FM-SGBEM described in the previous sections is now applied on a series of examples: a cube subjected to tensile load and fixed on the opposite face (Sec. 4.1), a crack in an unbounded medium (Sec. 4.2) and in a cylindrical solid (Sec. 4.4), and finally a $6 \times 6 \times 6$ array of cracks in an unbounded medium (Sec. 4.3). The results to follow were obtained on a OptiPlex 755 Desktop PC computer with a single $2.66 \mathrm{GHz}$ CPU and a 2GB RAM.

\subsection{Cube under uniaxial load}

This example involves a cube of unit side length, fixed along its bottom face and subjected to a unit tensile force density on its top face (Fig. 4). No exact solution is available for this problem. A FM-SGBEM solution has been computed, a FEM solution having also been performed for comparison purposes. Regular meshes of four-noded quadrilateral elements have been used, with the unknown traction field on the bottom face also modelled using this continuous interpolation (even though discontinuous tractions across elements could also have been adopted).

The FM-SGBEM solutions obtained for several mesh densities are compared on Figs. 5 to the reference solution for the displacement $u_{z}$ along a vertical edge and the stress $\sigma_{z z}$ on the bottom face. Unsurprisingly, the coarser meshes yield acceptable results for $u_{z}$ while $\sigma_{z z}$ requires finer meshes, especially near the edges.

The computing times for traditional SGBEM and FM-SGBEM are compared in Table 1, with the stopping criterion for GMRES defined in terms of a $10^{-5}$ relative tolerance. The CPU time per GMRES iteration are seen to follow the expected trends, namely a quadratic and linear dependence, respectively, with respect to the problem size $N$. The increasing relative efficiency of the FMSGBEM over the SGBEM is thus manifest. 

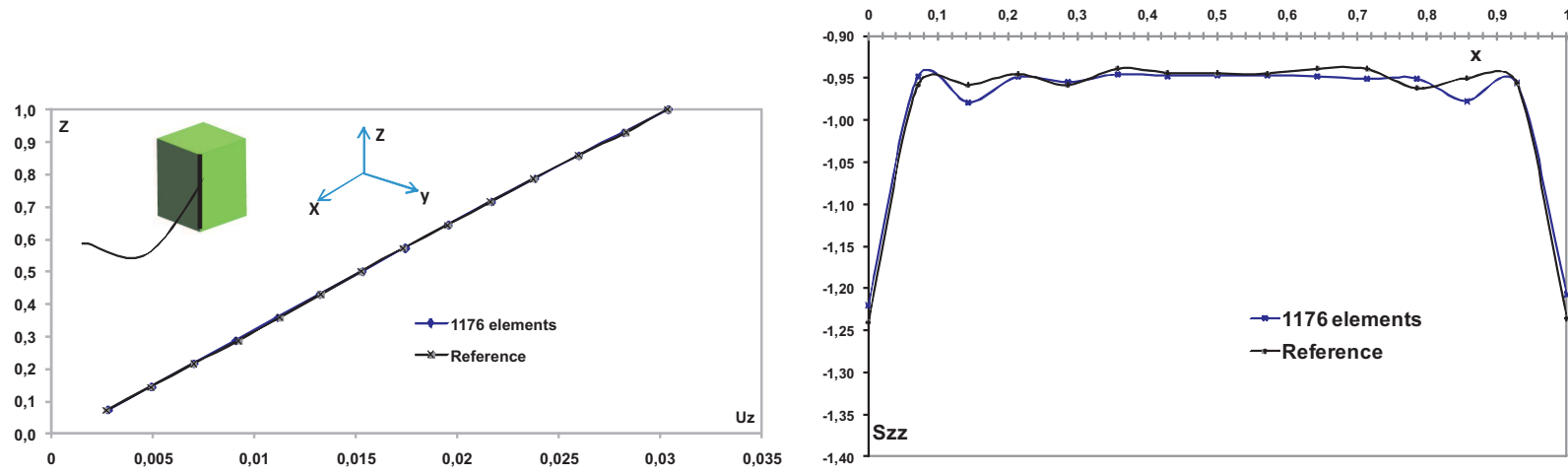

Figure 5: Clamped cube under uniaxial load: displacement $u_{z}$ along the vertical edge $x=y=1, z \in[0,1]$ (left) and stress $\sigma_{z z}$ evaluated along the line $x \in[0,1], y=0.5, z=0$ of the clamped face.

\subsection{Single penny-shaped crack in an unbounded body}

Then, a simple crack problem having a known exact solution is considered, namely that of a penny-shaped crack $S_{c}$ of radius $a$ and unit normal $\boldsymbol{n}=(0,0,1)$, embedded in an infinite isotropic elastic medium $(\nu=0.3, \mu=0.3846)$, opened by a uniform remote normal tension having the magnitude of $\sigma_{33}^{0}$ (see Fig. 6). For this particular situation, skipping all intermediate steps, the only unknown (namely the normal displacement discontinuity $\phi_{3}$ ) is governed by the variational traction equation on $S_{c}$ :

$$
\int_{S_{c}} \int_{S_{c}}\left[R \phi_{3}\right]_{q}(\boldsymbol{x}) B_{33 q s}(\boldsymbol{r})[R \widetilde{u}]_{s}(\tilde{\boldsymbol{x}}) \mathrm{d} S_{\tilde{x}} \mathrm{~d} S_{x}=\int_{S_{c}} \sigma_{33}^{0}(\boldsymbol{x}) \widetilde{\phi}_{3}(\boldsymbol{x}) \mathrm{d} S_{x}
$$

with $[R \phi]_{i q}$ relevant only with $i=3$ and thus reducing to $\left[R \phi_{3}\right]_{q}:=\delta_{s 2} \phi_{3,1}-\delta_{q 1} \phi_{3,2}$

Denoting by $r$ the distance from the crack center, the exact solution under remote tensile stress $\sigma_{33}^{0}$ reads [39]:

$$
\phi_{3}(\boldsymbol{x})=\frac{4(1-\nu)}{\pi \mu} \sqrt{a^{2}-r^{2}} \sigma_{33}^{0}, \quad K_{I}=\frac{2}{\pi} \sqrt{\pi a} \sigma_{33}^{0}
$$

\begin{tabular}{|c|c||c|c|c|c|c|c|}
\hline \multirow{2}{*}{ Mesh } & \multirow{2}{*}{$N$} & \multicolumn{2}{|c|}{ CPU per iteration (s) } & \multicolumn{2}{|c|}{ CPU total (s) } & \multirow{2}{*}{ iters } & $\bar{\ell}$ \\
\cline { 3 - 6 } & & SGBEM & FM-SGBEM & SGBEM & FM-SGBEM & & \\
\hline \hline 1 & 3,534 & 19 & 9 & 750 & 392 & 37 & 4 \\
\hline 2 & 8,718 & 118 & 16 & 5,551 & 745 & 45 & 4 \\
\hline 3 & 16,206 & 407 & 23.1 & 21,911 & 1,276 & 52 & 4 \\
\hline 4 & 25,998 & $1,356^{\star}$ & 29.4 & $60,564^{\star}$ & 1,913 & 59 & 4 \\
\hline 5 & 45,006 & $4,059^{\star}$ & 55.1 & $190,000^{\star}$ & 3,622 & 58 & 4 \\
\hline 6 & 180,006 & - & 92 & - & 24,588 & 284 & 5 \\
\hline 7 & 450,006 & - & 190 & - & 86,400 & 382 & 5 \\
\hline
\end{tabular}

Table 1: Clamped cube under uniaxial load: solution CPU times (per iteration, and overall) using either the SGBEM of FM-SGBEM, GMRES iteration counts and octree depth $\bar{\ell}$. The starred CPU times are extrapolations using the $O\left(N^{2}\right)$-per-iteration complexity 


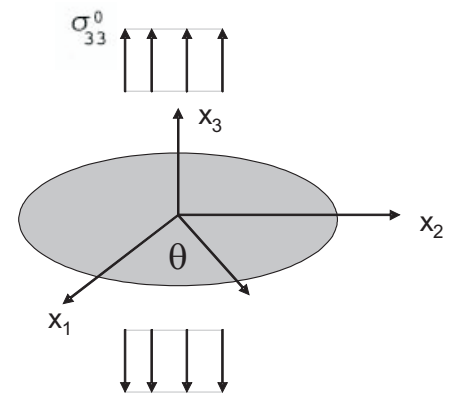

(a)

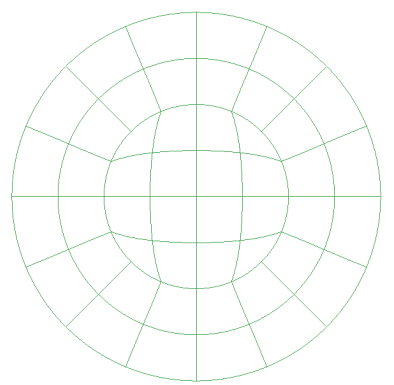

(b)

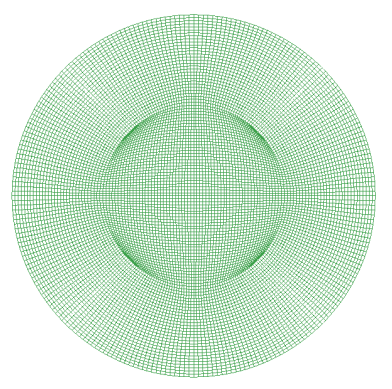

(c)

Figure 6: Single penny-shaped crack in an unbounded body: loading (a), mesh 1 (b) and mesh 6 (c).

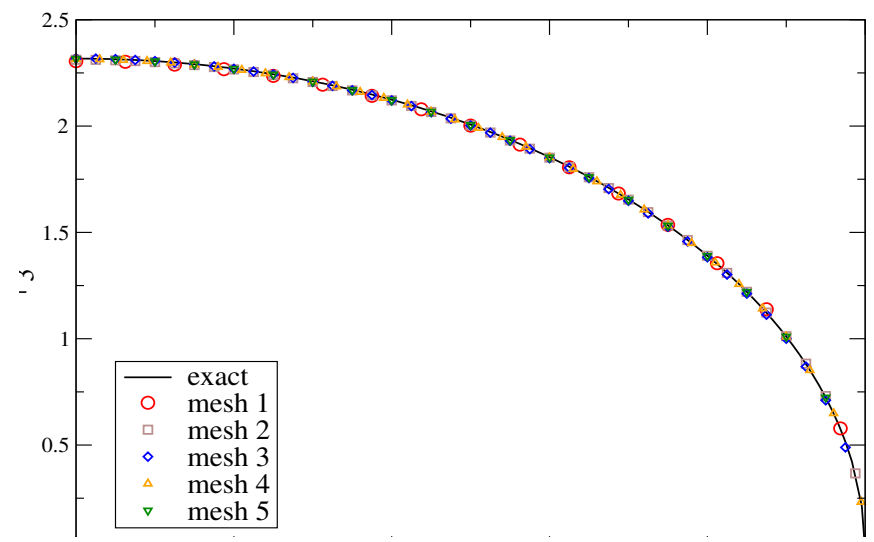

Figure 7: Single penny-shaped crack in an unbounded body: variation of $\phi_{3}$ along a crack radius, with meshes 1 to 5 as defined in Table 2

Five meshes, constituted of 8-noded quadrilateral elements and ranging from $N=1635$ to $N=$ 96483, have been used in this test (see Table 2).

Figure 7 shows the obtained nodal values of $\phi_{3}$ compared with the exact solution (32) along a radial line emanating from the crack center, while the relative $L^{2}$ errors on $\phi_{3}$ are given in Table 2.

\begin{tabular}{|c|c|c|c|c|c|c|}
\hline Mesh & Nodes & Elements & $N$ & \multicolumn{3}{|c|}{ rel. error } \\
\cline { 5 - 7 } & & & & $\phi_{3}$ & $K_{I}$ using (33) & $K_{I}$ using (34) \\
\hline 1 & 609 & 192 & 1,635 & $4.610^{-3}$ & $1.410^{-2}$ & $1.410^{-3}$ \\
2 & 3,681 & 1,200 & 10,563 & $1.710^{-3}$ & $4.610^{-3}$ & $9.310^{-4}$ \\
3 & 14,561 & 4,800 & 42,723 & $9.610^{-4}$ & $2.710^{-3}$ & $1.610^{-3}$ \\
4 & 22,701 & 7,500 & 66,903 & $8.410^{-4}$ & $2.410^{-3}$ & $1.710^{-3}$ \\
5 & 32,641 & 10,800 & 96,483 & $6.710^{-4}$ & $2.310^{-3}$ & $1.710^{-3}$ \\
\hline
\end{tabular}

Table 2: Single penny-shaped crack in an unbounded body: mesh parameters. 


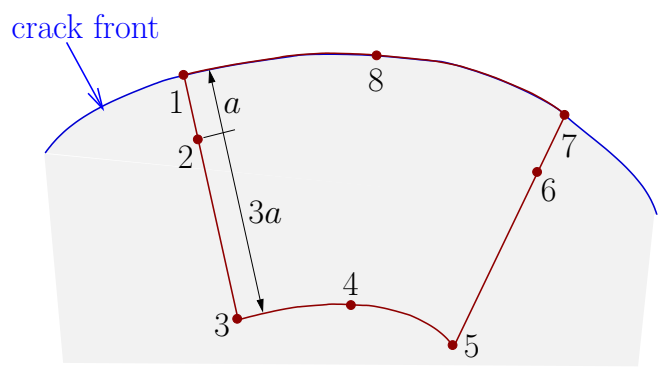

Figure 8: Quarter-point 8-noded quadrilateral crack front element

\begin{tabular}{|c||c|c|c|c|c|}
\hline \multirow{2}{*}{ Mesh } & $\ell$ & \multicolumn{2}{|c|}{ CPU/iter (s) } & iters & $\begin{array}{c}\text { CPU total (s) } \\
\text { (FM-SGBEM) }\end{array}$ \\
\cline { 3 - 4 } & & SGBEM & FM-SGBEM & & 53 \\
\hline 2 & 3 & 1 & 0,7 & 18 & 376 \\
3 & 4 & 42.5 & 5.4 & 26 & 1,207 \\
4 & 6 & 695 & 11.8 & 97 & 2,404 \\
5 & 6 & 1,700 & 14.9 & 150 & 6,012 \\
6 & 6 & 3,540 & 24,3 & 237 & \\
\hline
\end{tabular}

Table 3: Single penny-shaped crack in an unbounded body: octree depths $\bar{\ell}$, CPU time per iteration for SGBEM and FM-SGBEM, GMRES iteration counts, and total FM-SGBEM solution times

The elements adjacent to the crack front $\partial S_{c}$ are special 8-noded quadrilateral elements where the middle nodes on the edges perpendicular to $\partial S_{c}$ are located one-quarter of the edge length away from $\partial S_{c}$ [3]. This feature allows to approximate the $d^{1 / 2}$ behaviour of $\phi$ near $\partial S_{c}$. The stress intensity factor at node 1 (with the numbering convention of Fig. 8) can then be evaluated from the nodal opening displacements at nodes 1 and 2 by :

$$
K_{I}^{1}=\lim _{d \rightarrow 0} \frac{\mu}{4(1-\nu)}\left(\frac{2 \pi}{d}\right)^{1 / 2} \phi_{3} \approx \frac{\mu}{4(1-\nu)}\left(\frac{2 \pi}{d}\right)^{1 / 2}\left[2 \phi_{3}^{(2)}-\frac{1}{2} \phi_{3}^{(3)}\right]
$$

The following alternative approximation formula is also available for $K_{I}$ :

$$
K_{I}^{1} \approx \frac{\mu}{4(1-\nu)}\left(\frac{2 \pi}{d}\right)^{1 / 2} \phi_{3}^{(2)}
$$

Table 2 shows the relative errors for $K_{I}$ evaluated using either (33) or (34). The latter being seen to provide more accurate results.

Table 3 reports the CPU time per iteration incurred by both the FM-SGBEM and the traditional SGBEM for the complete solution for meshes 1 to 6 (using GMRES for both approaches), together with the octree depths $\bar{\ell}$, GMRES iteration counts, and total FM-SGBEM solution times. The FM-SGBEM is always faster than the conventional SGBEM, and the complexity of the former is, as expected, lower.

\subsection{Crack array in an unbounded body}

A multi-crack configuration is now considered, where an unbounded elastic medium (with again $\nu=0.3, \mu=0.3846$ ) contains an array of $M \times M \times M=M^{3}$ penny-shaped cracks of identical radius $a$ whose centers are located at the vertices of a regular cubic grid (with space step $4 a$ ) 


\begin{tabular}{|c||c|c|c|c|}
\hline array & DOFs & CPU/iter $(\mathrm{s})$ & iters & CPU total $(\mathrm{s})$ \\
\hline $6 \times 6 \times 6$ & 83,592 & 121,4 & 17 & 3,738 \\
$14 \times 14 \times 14$ & $1,061,928$ & 1,542 & 18 & 45,695 \\
\hline
\end{tabular}

Table 4: Crack array in an unbounded body: problem sizes and runtime results

and whose normals are randomly chosen. Two arrays have been considered, with respectively $M=6$ and $M=14$; the latter array involves over one million DOFs. A uniform remote tensile loading $\sigma_{33}^{0}$ is applied to the system. Each crack is modelled using the 387-DOF mesh shown in Fig. 9 constituted of 48 eight-noded quadratic quadrilateral elements. The mesh and normalized computed crack opening displacement values $\phi_{3} /\left(a \sigma_{33}^{0}\right)$ are shown for the whole crack array on Fig. 10 for $M=6$. The computational times and iteration counts are shown in Table 4. Finally, to illustrate remarks made on memory requirements at the end of Sec. 3.1, running the $M=14$ million-DOF example required roughly $6 \mathrm{~Gb}$ of memory (5Gb for storing the sparse near matrix, and $1 \mathrm{~Gb}$ as workspace for GMRES). For comparison purposes, using the standard SGBEM, the same amount of memory would allow to hold the symmetric fully-populated influence matrix $[B]$ with $N \approx 40,000$.

\subsection{Single crack in a bounded body}

Finally, the case of a cylindrical finite elastic body $\Omega$ (of axis $O x_{3}$, length $H$ and radius $R$, and with again $\nu=0.3, \mu=0.3846)$ containing a penny-shaped crack $S_{c}$ with radius $a$ and unit normal $\boldsymbol{n}=(0,0,1)$ (Fig. 11) is considered. Uniform tensile stresses are applied on the two end surfaces of the cylinder, while the other surfaces are stress-free. The geometric characteristics are set to $H=60 a$ and $R=10 a$, making this problem almost identical to that of a crack in an infinite cylinder. Three meshes A, B and C (Fig. 12), featuring 2,121, 8553 and 46,569 DOFs and 48, 192 and 1200 elements on the crack surface, respectively, were used (see Table 5 for the main mesh parameters, GMRES iteration counts, overall CPU times recorded, and octree depths $\bar{\ell}$ ).

In this particular case, the governing SGBIE formulation is obtained by setting $S_{u}=\emptyset, S_{T}=S$ in (16) and definitions (7), (9) to obtain

$$
\text { Find }(\boldsymbol{u}, \phi) \in \mathcal{V}_{u} \times \mathcal{V}_{c},\left\{\begin{array}{l}
\mathcal{B}_{u u}(\boldsymbol{u}, \widetilde{\boldsymbol{u}})+\mathcal{B}_{\phi u}(\phi, \widetilde{\boldsymbol{u}})=\mathcal{F}_{u}(\widetilde{\boldsymbol{u}}) \\
\mathcal{B}_{u \phi}(\boldsymbol{u}, \widetilde{\boldsymbol{\phi}})+\mathcal{B}_{\phi \phi}(\phi, \widetilde{\boldsymbol{\phi}})=\mathcal{F}_{\phi}(\widetilde{\boldsymbol{\phi}})
\end{array} \quad \forall(\widetilde{\boldsymbol{u}}, \widetilde{\boldsymbol{\phi}}) \in \mathcal{V}_{u} \times \mathcal{V}_{c}\right.
$$

Figure 13 compares the obtained nodal values of the normal crack opening displacement along a radial line to the solution (32), in terms of their values and relative errors. Table 5 also shows the relative errors for the SIFs, evaluated using either (33) or (34).

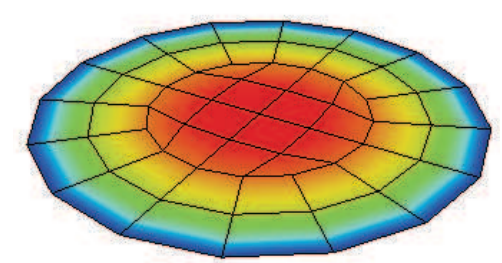

Figure 9: Crack array in an unbounded body: 387-DOF BE mesh of a single crack 


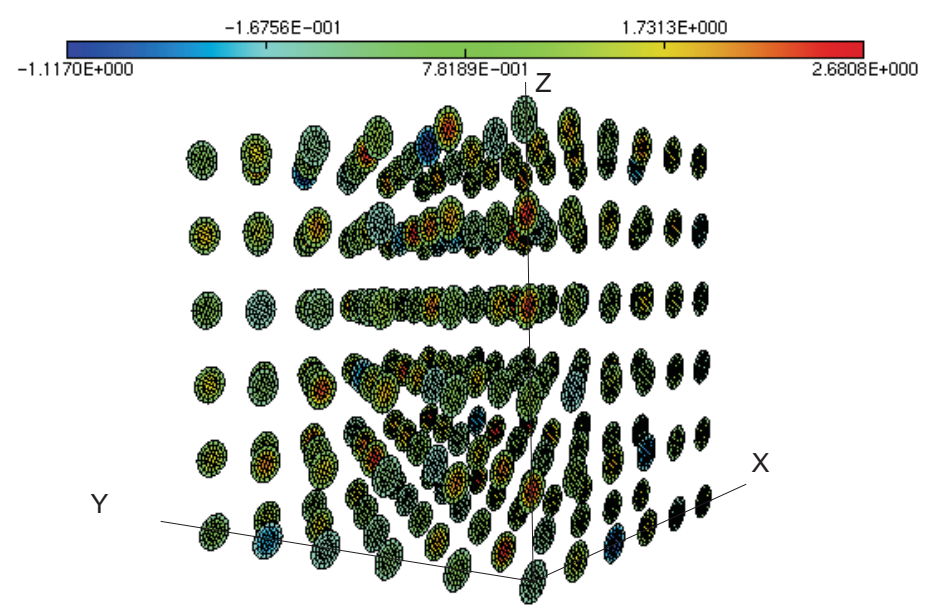

Figure 10: Crack array in an unbounded body: crack opening displacements

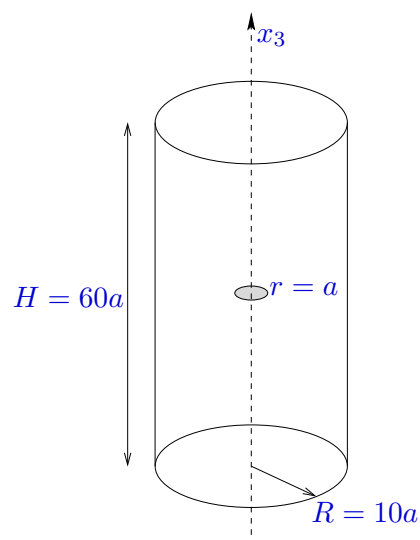

Figure 11: Cylinder containing a circular surface crack

\begin{tabular}{|c|c|c|c|c|c|c|c|c|c|c|c|}
\hline \multirow[t]{2}{*}{ mesh } & \multicolumn{2}{|c|}{ nodes } & \multicolumn{2}{|c|}{ elements } & \multicolumn{2}{|c|}{$N$} & \multirow[t]{2}{*}{ iters } & \multirow{2}{*}{$\begin{array}{c}\mathrm{CPU} \\
(\mathrm{s})\end{array}$} & \multirow[t]{2}{*}{$\bar{\ell}$} & \multicolumn{2}{|c|}{ rel. error on $K_{I}$} \\
\hline & crack & overall & crack & overall & crack & overall & & & & using (33) & using (34) \\
\hline $\mathrm{A}$ & 161 & 739 & 48 & 240 & 387 & 2,121 & 19 & 63 & 7 & $4.510^{-2}$ & $1.410^{-2}$ \\
\hline B & 609 & 2,915 & 192 & 960 & 1,639 & 8,553 & 25 & 431 & 7 & $2.310^{-2}$ & $5.610^{-3}$ \\
\hline $\mathrm{C}$ & 3,681 & 15,683 & 1,200 & 5,200 & 10,563 & 46,569 & 37 & 3,266 & 9 & $8.910^{-3}$ & $2.810^{-3}$ \\
\hline
\end{tabular}

Table 5: Circular crack in a cylinder: mesh parameters, GMRES iteration counts, overall CPU times recorded, octree depths $\bar{\ell}$, and relative errors on $K_{I}$.

\subsection{Multiple crack in a bounded body}

Finally, the case of a box-shaped elastic body (of size $A \times A \times B$, with $B=2 A=50 a$ ) containing an array of $M \times M \times M=M^{3}$ penny-shaped cracks of identical radius $a$ whose centers are located at the vertices of a regular cubic grid (whose center coincides with the box center, and with horizontal spacing $\alpha a$ and vertical spacing $\beta a$ ) and with randomly chosen normals. The Poisson's ratio is set to $\nu=0.3$. A uniform tensile load is applied on the top surface, while the bottom surface is clamped. Three arrays have been considered, with $(M, \alpha)$ as given in Table 6 . The external 

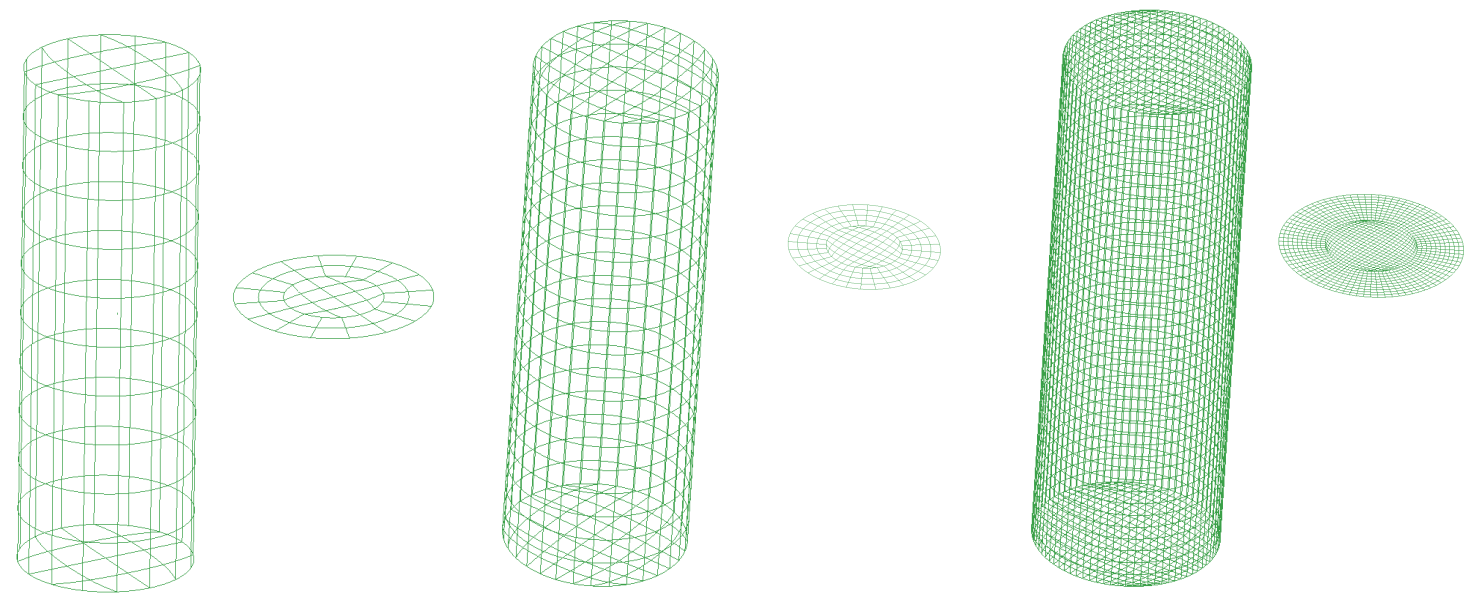

Figure 12: Circular crack in a cylinder: meshes A (left), B (middle) and C (right)

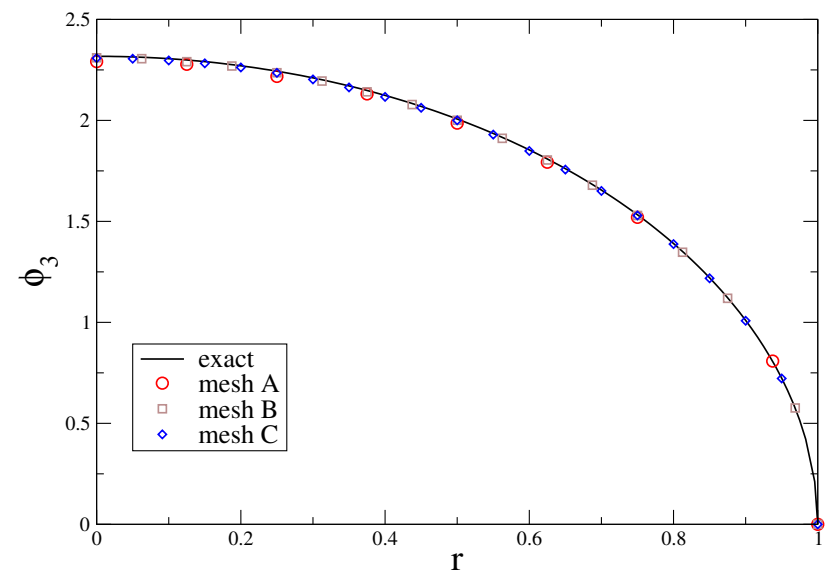

Figure 13: Circular crack in a cylinder: numerical results for $\phi_{3}$, with meshes A, B, C as defined in Table 5.

\begin{tabular}{|c|c|c|c|c|c|c|c|c|c|}
\hline \multicolumn{2}{|c|}{ array } & \multicolumn{2}{|c|}{ nodes } & \multicolumn{2}{c|}{ elements } & \multicolumn{2}{|c|}{$N$} & iters & CPU \\
\cline { 1 - 8 }$M$ & $\alpha$ & cracks & overall & crack & overall & crack & overall & & $(\mathrm{s})$ \\
\hline 4 & 6 & 10,304 & 10,906 & 3,072 & 3,672 & 24,768 & 26,574 & 38 & 4,021 \\
6 & 6 & 33,166 & 33,768 & 10,368 & 10,968 & 83,592 & 85,398 & 36 & 11,688 \\
8 & 4 & 82,432 & 83,034 & 24,576 & 25,176 & 198,144 & 199,950 & 52 & 35,665 \\
\hline
\end{tabular}

Table 6: Multiple crack in a bounded body: mesh parameters, GMRES iteration counts, overall CPU times recorded.

surface is meshed with $10 \times 10$ four-noded elements on each face, while each crack comprises 48 eight-noded elements, 161 nodes and 387 DOFs. The recorded CPU times and iteration counts are reported, together with the main characteristics of the mesh, in Table 6 . The CPU times grow roughly linearly with the problem size, as expected. 


\section{Conclusion}

In this article, the formulation and implementation of a multi-level fast multipole SGBEM (FMSGBEM) for elastic solid with cracks, applicable for arbitrary geometries and boundary conditions, was presented. Numerical results on test problems on BE models involving up to $10^{6}$ unknowns, featuring a clamped cube, single or multiple cracks in an unbounded medium, a cracked cylindrical solid, and a multi-cracked box-shaped solid, were discussed. They follow the predicted desirable trends of the elastostatic FM-SGBEM, such as a $O(N)$ complexity per iteration. Future work includes optimizing the implementation and applying the resulting code to rock materials with complex fracture networks.

\section{Appendix A. Fast Multipole Method: useful formulae}

This appendix provides the formulae for the practical computation of the solid harmonics $R_{n, m}(\boldsymbol{y})$ and $S_{n, m}(\boldsymbol{y})$ and of the derivatives of the $R_{n, m}$ using only the Cartesian coordinates of generic the argument $\boldsymbol{y}$, after references $[45,46]$ to which the reader is referred for more details. First, the evaluation of $R_{n, m}(\boldsymbol{y})$ and $S_{n, m}(\boldsymbol{y})$ is performed as follows:

(a) The $R_{n, m}(\boldsymbol{y})$ are computed recursively by setting $R_{0,0}(\boldsymbol{y})=1$ and using

$$
\begin{aligned}
& R_{n+1, n+1}(\boldsymbol{y})=\frac{y_{1}+\mathrm{i} y_{2}}{2(n+1)} R_{n, n}(\boldsymbol{y}) \\
& \left((n+1)^{2}-m^{2}\right) R_{n+1, m}(\boldsymbol{y})-(2 n+1) y_{3} R_{n, m}(\boldsymbol{y})+\|\boldsymbol{y}\|^{2} R_{n-1, m}(\boldsymbol{y})=0
\end{aligned}
$$

(b) The $S_{n, m}(\boldsymbol{y})$ are computed recursively by setting $S_{0,0}(\boldsymbol{y})=1 /\|\boldsymbol{y}\|$ and using

$$
\begin{aligned}
& S_{n+1, n+1}(\boldsymbol{y})=\frac{(2 n+1)\left(y_{1}+\mathrm{i} y_{2}\right)}{\|\boldsymbol{y}\|^{2}} R_{n, n}(\boldsymbol{y}) \\
& \|\boldsymbol{y}\|^{2} S_{n+1, m}(\boldsymbol{y})-(2 n+1) y_{3} S_{n, m}(\boldsymbol{y})+\left(n^{2}-m^{2}\right) S_{n-1, m}(\boldsymbol{y})=0
\end{aligned}
$$

(c) Finally, $R_{n, m}(\boldsymbol{y})$ and $S_{n, m}(\boldsymbol{y})$ for negative values of $m$ are computed via the identities

$$
\begin{aligned}
R_{n,-m}(\boldsymbol{y}) & =(-1)^{m} \overline{R_{n, m}(\boldsymbol{y})} \\
S_{n,-m}(\boldsymbol{y}) & =(-1)^{m} \overline{S_{n, m}(\boldsymbol{y})}
\end{aligned}
$$

Identities (A.2) and (A.4) are used with $n \geq m$. In addition, the derivatives of the $R_{n, m}$ are computed via

$$
\begin{aligned}
\frac{\partial}{\partial y_{1}} R_{n, m}(\boldsymbol{y}) & =\frac{1}{2}\left(R_{n-1, m-1}-R_{n-1, m+1}\right)(\boldsymbol{y}) \\
\frac{\partial}{\partial y_{2}} R_{n, m}(\boldsymbol{y}) & =\frac{i}{2}\left(R_{n-1, m-1}+R_{n-1, m+1}\right)(\boldsymbol{y}) \\
\frac{\partial}{\partial y_{3}} R_{n, m}(\boldsymbol{y}) & =R_{n-1, m}(\boldsymbol{y})
\end{aligned}
$$




\section{References}

[1] Aliabadi, M. H. Boundary element formulations in fracture mechanics. Appl. Mech. Rev., 50:83-96 (1997).

[2] Bapat, M. S., Shen, L., Liu, Y. J. Adaptive fast multipole boundary element method for three-dimensional half-space acoustic wave problems. Eng. Anal. Bound. Elem., 33:1113-1123 (2009).

[3] Barsoum, R. S. On the use of isoparametric finite element in linear fracture mechanics. Int. J. Num. Meth. Eng., 10:25-37 (1976).

[4] Bécache, E., Nedelec, J.-C., Nishimura, N. Regularization in 3D for anisotropic elastodynamic crack and obstacle problems. J. Elast., 31:25-46 (1993).

[5] Beer, G., Smith, I., Duenser, C. The boundary element method with programming. Springer (2008).

[6] Bonnet, M. Regularized direct and indirect symmetric variational BIE formulations for three-dimensional elasticity. Eng. Anal. Bound. Elem., 15:93-102 (1995).

[7] Bonnet, M. Boundary integral equation methods for solids and fluids. John Wiley \& Sons (1999).

[8] Bonnet, M. Stability of crack fronts under Griffith criterion: a computational approach using integral equations and domain derivatives of potential energy. Comp. Meth. Appl. Mech. Eng., 173:337-364 (1999).

[9] Bonnet, M., Maier, G., Polizzotto, C. Symmetric Galerkin boundary element method. Appl. Mech. Rev., 51:669-704 (1998).

[10] Brebbia, C. A., Telles, J. C. F., Wrobel, L. C. Boundary element techniques. Springer-Verlag (1984).

[11] Brunner, D., Junge, M., L., Gaul. A comparison of FE-BE coupling schemes for large-scale problems with fluid-structure interaction. Int. J. Num. Meth. Eng., 77:664-688 (2009).

[12] Bui, H. D. An integral equation method for solving the problem of a plane crack of arbitary shape. J. Mech. Phys. Solids, 25:29-39 (1977).

[13] Chaillat, S., Bonnet, M., Semblat, J. F. A multi-level fast multipole BEM for 3-D elastodynamics in the frequency domain. Comp. Meth. Appl. Mech. Eng., 197:4233-4249 (2008).

[14] Chen, Y. H., Chew, W. C., Zeroug, S. Fast multipole method as an efficient solver for 2D elastic wave surface integral equations. Comp. Mech., 20:495-506 (1997).

[15] Chew, W. C. et al. Fast integral equation solvers in computational electromagnetics of complex structures. Eng. Anal. Bound. Elem., 27:803-823 (2003).

[16] Cruse, T. A. Numerical solutions in three-dimensional elastostatics. Int. J. Solids Struct., 5:1259-1274 (1969).

[17] Cruse, T. A. Boundary Element Analysis in Computational Fracture Mechanics. Kluwer Academic Publishers (1988).

[18] Frangi, A., Di Gioia, A.. Multipole BEM for the evaluation of damping forces on MEMS. Comp. Mech., 37:24-31 (2005).

[19] Frangi, A., Novati, G., Springhetti, R., Rovizzi, M. 3D fracture analysis by the symmetric Galerkin BEM. Comp. Mech., 28:220-232 (2001).

[20] Gomez, J. E., Power, H. A multipole direct and indirect BEM for 2D cavity flow at low Reynolds number. Eng. Anal. Bound. Elem., 19:17-31 (1997).

[21] Gray, L. J., Chinta, B., Kane, J. H. Symmetric Galerkin fracture analysis. Eng. Anal. Bound. Elem., 15:103-109 (1995).

[22] Gray, L. J., Paulino, G. H. Symmetric Galerkin boundary integral formulation for interface and multi-zone problems. Int. J. Num. Meth. Eng., 40:3085-3101 (1997).

[23] Greengard, L., Rokhlin, V. A fast algorithm for particle simulations. J. Comp. Phys., 73:325-348 (1987).

[24] Greengard, L., Rokhlin, V. A new version of the fast multipole method for the Laplace equation in three dimensions. Acta Numerica, 6:229-270 (1997).

[25] Hackbusch, W., Nowak, Z. P. On the fast matrix multiplication in the boundary element method by panel clustering. Numer. Math., 54:463-491 (1989).

[26] Kurz, S., Rain, O., Rjasanow, S. The adaptive cross approximation technique for the 3D boundary element method. IEEE Transactions on Magnetics, 38:421-424 (2002).

[27] Lai, Y.-S., Rodin, G. J. Fast boundary element method for three-dimensional solids comntaining many cracks. Eng. Anal. Bound. Elem., 27:845-852 (2003).

[28] Li, S., Mear, M. E., Xiao, L. Symmetric weak-form integral equation method for three-dimensional fracture analysis. Comp. Meth. Appl. Mech. Eng., 151:435-459 (1998).

[29] Margonari, M., Bonnet, M. Fast multipole method applied to the coupling of elastostatic BEM with FEM. Comput. Struct., 83:700-717 (2005).

[30] Mi, Y., Aliabadi, M. H. Dual boundary element method for three-dimensional fracture mechanics analysis. Eng. Anal. Bound. Elem., 10:161-171 (1992).

[31] Nedelec, J. C. Integral equations with non integrable kernels. Integ. Equ. Oper. Theory, 5:562-572 (1982). 
[32] Nishimura, N. Fast multipole accelerated boundary integral equation methods. Appl. Mech. Rev., 55:299-324 (2002).

[33] Nishimura, N., Yoshida, S., Kobayashi, S. A fast multipole boundary integral equation method for crack problems in 3D. Eng. Anal. Bound. Elem., 23:97-105 (1999).

[34] Of, G., Steinbach, O., Wendland, W. L. The fast multipole method for the symmetric boundary integral formulation. IMA J. Num. Anal., 26:272-296 (2006).

[35] Rizzo, F. J. An integral equation approach to boundary value problems of classical elastostatics. Quart. Appl. Math., 25:83-95 (1967).

[36] Rokhlin, V. Rapid solution of integral equations of classical potential theory. J. Comp. Phys., 60:187-207 (1985).

[37] Saad, Y., Schultz, M.H. GMRES: a generalized minimal residual algorithm for solving nonsymmetric linear systems. SIAM J. Sci. Stat. Comput., 7:856-869 (1986).

[38] Sirtori, S., Maier, G., Novati, G., Miccoli, S. A Galerkin symmetric boundary element method in elasticity: formulation and implementation. Int. J. Num. Meth. Eng., 35:255-282 (1992).

[39] Sneddon, I. N., Lowengrub, M. Crack problems in the classical theory of elasticity. Wiley (1969).

[40] Sylvand, G. La méthode multipôle rapide en électromagnétisme : performances, parallélisation, applications. Ph.D. thesis, Ecole Nationale des Ponts et Chaussées, Noisy le Grand, France (2002).

[41] Takahashi, T., Nishimura, N., Kobayashi, S. A fast BIEM for three-dimensional elastodynamics in time domain. Eng. Anal. Bound. Elem., 28:165-180 (2004).

[42] Tausch, J. Sparse BEM for potential theory and Stokes flow using variable order wavelets. Comp. Mech., 32:312-3185 (2003).

[43] Yasuda, Y., Sakuma, T. A technique for plane-symmetric sound field analysis in the fast multipole boundary element method. J. Comput. Acoust., 13:71-85 (2005).

[44] Ying, L., Biros, G., Zorin, D. A kernel-independent adaptive fast multipole in two and three dimensions. J. Comp. Phys., 196:591-626 (2004).

[45] Yoshida, K.I. Applications of fast multipole method to boundary integral equation method. Ph.D. thesis, University of Kyoto (2001).

[46] Yoshida, S., Nishimura, N., Kobayashi, S. Application of fast multipole Galerkin boundary integral equation method to elastostatic crack problems in 3D. Int. J. Num. Meth. Eng., 50:525-547 (2001). 